\title{
Editorial: How Digital Innovators Achieve Customer Value
}

\author{
Christof Gellweiler ${ }^{1}$, Lakshman Krishnamurthi ${ }^{2}$ \\ ${ }^{1}$ WHU - Otto Beisheim School of Management, Vallendar, Germany \\ christof.gellweiler@whu.edu \\ ${ }_{2}$ Northwestern University, Kellogg School of Management, Marketing, Evanston, IL, The United States of America \\ laksh@kellogg.northwestern.edu \\ January 2020
}

\section{Introduction}

This editorial presents special features of digitization in context with innovation. Digital innovators go beyond new product development and emerging technologies. They change business systems and make use of sociocultural trends to deliver value. New technologies and creative business models provide essential ingredients for digital innovation. However, crucial are the combinations of these innovations in terms of the advantages that customers perceive. The conventional e-commerce model should integrate more digital innovations by continuously collecting and evaluating data from intelligent, sensor-equipped objects to enhance customer experience. Additional channels, new points of presence, and convenient payment methods are components for designing promising digital services. In this article, we provide a set of propositions derived from prominent cases in practice. We seek to inspire exploratory studies in the young research field of digital innovation.

Digitization of the physical world has tremendous impact on numerous industries; digitization enables new services and changes the rules of the competition. Innovations, in general, concern changes driven by digital technologies in product features, distribution, communication, pricing, or product positioning, with the goal of providing benefits to customers, either economic, functional, or psychological [34]. Digital strategies, and the corresponding capabilities, involve offering services in which customers' needs are central [23]. Moreover, digitization allows companies to gain more control of their processes, thus increasing the firms' operational effectiveness [6], [40]. However, the core and the consequences of digitization are still unclear, which leads to noticeable uncertainty within various business areas.

We attempt to outline the essence of digitization and innovation, focusing on customer value creation. We briefly describe the major aspects of digital innovation and discuss selected business cases. Next, we sketch the traditional e-commerce model and raise questions about enhancements in e-commerce by digital innovations. In the conclusion, we summarize the key points and indicate limitations.

\section{Digital Innovations Go Beyond New Product Development}

Digital innovators, which are companies that successfully seize digital opportunities, provide unique combinations of conventional and digital factors for innovations. Digital innovators focus on customer value, especially cost benefits and the customer experience, applying established marketing practices and procedures for the development of new products or services [43].

Common approaches for product and service introductions are employed by digital innovators; new ideas are collected and screened, feasibility and business chances are analyzed, and products are developed and tested. Likewise, digital innovators involve customers in researching their needs, for example, by proof-of-concept [44]. New products and services typically improve performance compared to the predecessor product and existing competitors' offerings. Reliability, operator comfort, flexibility, and ease of maintenance are some examples of classic performance factors that advance with new products [3]. These aspects and other marketing ground rules constitute the common denominator of successful new products and services.

Digital innovators build on the same foundation, but their particularities arise from other factors, which are skillfully utilized. These digital innovation factors are (i) interconnection of people and assets via public and private networks; (ii) application of human-imitating technologies, particularly sensors and artificial intelligence; (iii) utilization of social trends; and (iv) creation or change of business systems.

Figure 1 illustrates the relevant components. Intelligent and sensor-equipped objects connected via access and backbone networks constitute the center of digital innovation. Changing the architecture of business systems and taking advantage of social trends are basic approaches. The ultimate goal is to create value for customers through various combinations. 


\section{Customer value disciplines and benefits}

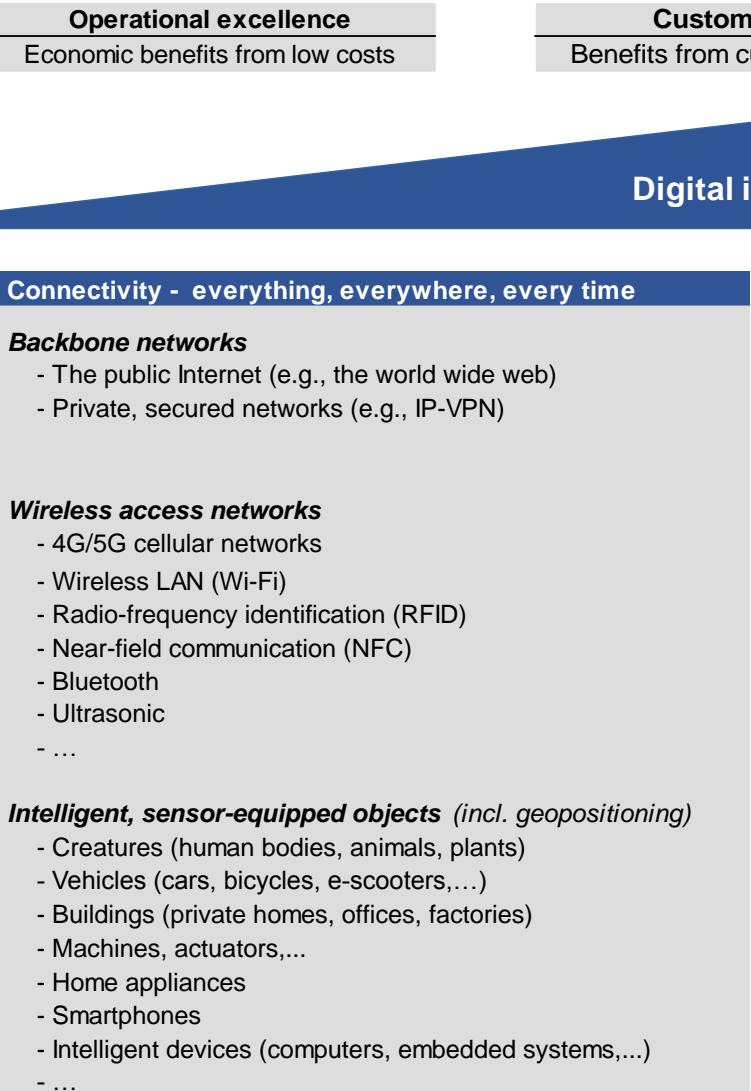

Change in business systems
Adding channels / including intermediaries
- Agents or brokers for promotion and selling
- Finance and insurance companies
- Distributors or value-adding resellers
- Other e-commerce firms
Points of presence at department stores, hypermarkets,
specialty stores, convenience stores, public places,...
Value capture
- Advertising
- Fees for usage and subscription
- License fees from franchising
- Charges for brokerage
- Credit cards
- Digital wallets, touch payment systems
Sociocultural trends
- Data permissiveness
- Chattiness on social media
- Sharing economy
- Health awareness
- Environmental care

Figure 1: The ingredients of digital innovation for customer value

(Source: Authors' work)

\section{The Role of Networks: Connectivity of Everything, Everywhere, Every Time}

Networks are the centerpieces of digital technologies [7]. Networks are mandatory for digital innovation to move data, which is generated and evaluated for value creation, between endpoints (e.g., clients, servers, and smart devices) [13]. The Internet connects to consumers and facilitates customer involvement in product innovations [35]. The Internet also provides transportation services between elements of the physical world, that is, things like vehicles, machines, buildings, animals, human bodies, and computing devices. With the aid of the Internet, these things send, receive, and process data, independent of time and location [17]. However, the common notion of the Internet of Things is inaccurate and misleading; private networks and access networks coexist and complement the public Internet for digital innovations. For confidential and business-critical applications, encrypted virtual private networks (VPN) form appropriate data highways between enterprise locations, the labor force, and associated business partners. Access network technologies bridge user endpoints to the public Internet or to private networks. In addition to well-known wireless access technologies, such as fourth-generation cellular networks (4G), near-field communication (NFC), or Wi-Fi, new developments are expected in the market (e.g., fifth-generation cellular networks (5G), low-power wide area networks [31], and ultrasonic solutions).

\section{Technological Enablers of Digital Innovation: Sensors and Artificial Intelligence}

The function of sensor devices, namely, measurement and analogue-to-digital conversion, and their rapid progression in recent years, provided the popular term digitization, and its literal derivatives, such as digitalization and digital transformation [5]. Miniaturization, that is, the integration of semi-conductors in smart devices, is progressing; it provides higher capacities for processing and storing data in small dimensions. These capacities allow high-performance computing of sensor-collected data, not only in smartphones or watches but also in tiny embedded systems, such as glasses, for workers in factories to scan serial numbers [38], or medical applications, for monitoring of vital signs. Sensor technology is one of the key drivers of digital innovations, because it allows translation of physical or chemical information from assets, living things, plants, and environments into a code that connected electronic devices can process at a distance. Sensors in various smart devices can measure local temperature, barometric pressure, humidity, brightness, and substances for remote operation. The application of 
sensors combined with geopositioning or audiovisual functions provides outstanding opportunities for the creation of useful digital services.

Augmented reality provides further options for novel experiences. It widens the user's perception by combining reality with electronic visualizations. Filtered information from sensors can be assimilated within augmented reality systems. Human capabilities from own sensory organs are extended by artificial sensors that can be located anyplace in the world, if they are connected via networks. These digital technologies enable innovations, and open up new perspectives for companies on established processes and current operations [26] p. 74.

Artificial intelligence (Al) is a complementary driver for pioneering digital solutions; it strives to replace human cognitive abilities, like learning and thinking, with machines. Embedded intelligence enables processes to optimize themselves [18]. The integration of Al with emulated human organs (e.g., cameras or sensors) and human motion represents the most challenging discipline for digital innovation, which is robotics, referring to the simulation of human beings. The addition of human-machine interfaces, such as speech recognition, allows intuitive instructable robots.

\section{Sociocultural Trends Drive Digital Innovation}

Several sociocultural trends drive digital innovation. The major tendencies are related to the decreased sensitivity of individuals regarding data protection, and the idea of asset-sharing. Social media has become one of the main influences of business and personal lives [33]. Customers' willingness to leave their personal data open to external software firms has greatly increased in recent years. Today, users share private data on social networks; for example, users chat intensively about personal topics and post private data. Furthermore, users allow mobile applications to process biometric, medical, and geographic location data, without being certain how these data are secured and protected from misuse. Regardless of whether psychologists specify this behavior as trust, thoughtlessness, or habit, it is usual and widespread. Erosion of privacy is a social phenomenon across multiple cultures around the globe.

This phenomenon does not stop at willingly sharing data across social networks. In addition, people permanently carry their smart equipment close to their bodies. Digital innovators fully exploit these actualities. Connected peripherals, such as watches, contain sensors that provide digital information on health conditions or on the user's physical environment. Therefore, digital firms can gather and evaluate highly sensitive personal data from users via smartphones-every time and everywhere. Installation and usage of value-adding applications on connected devices by users are the key to digital innovators' success. The readiness of a user to allow access to his or her data depends on the value that he or she perceives from the smartphone application.

Another path-breaking development in societies of wealthy countries is asset-sharing. An increasing number of people prefer to jointly use an asset rather than to exclusively own it (e.g., vehicles and work/living spaces). The socalled sharing economy provides financial benefits to asset owners and asset users through higher utilization [16]. The Internet is the medium for organizing reservations for shared assets and the associated monetary flows.

\section{Digital Innovators Change Business Models}

Innovation of business models is crucial for creating value [25]. The customer value proposition is an essential element of business models [14], especially in relation to the ways value is delivered and captured. Innovators must identify the market and analyze desired benefits for customers that drive willingness to pay [30]; these benefits signify the innovator's value proposition, more than the newness of the products or technologies [29] pp. $226-227$. Thus, benefits and values must be analyzed before the product is designed. Customer values stem from three types of benefits: superior product or service functions, low cost, or relationships [42]. Digital innovators should have in mind the economic benefits of low cost (value discipline: operational excellence), as well as the benefits from relationships, for example, brand image and customer experience (value discipline: customer intimacy). Economic benefits from low costs have two manifestations. Customers either buy a product or service for a lower price for the same quality as competitive offerings, or they receive more quality for the same price as competitive products or services [11]. However, in the digital age, the customer experience is the most significant benefit [41] p. 8.

Digital innovators may base their value propositions on the implementation of complementary marketing channels. The Internet and associated networks are foundational, but not exclusive, channels to customers. Supplementary channels extend market access, make the products and services more available for use, and generate additional customer value [8] p. 3. Value delivery via the Internet in combination with novel physical points of presence (e.g., smart retail stores) also increases differentiation from competitors. Presence at locations is a firm's specific asset; it enhances delivery of goods and increases customer value [11] p. 44. However, multiple channels must be carefully integrated to optimize the customer experience; important is a "single unified view of the customer" [27] p. 172.

Inclusion of intermediaries increase service outputs. Intermediaries might be retailers or firms participating in special flows, such as promotion, ordering, physical shipment, or payment [8] pp. 15-16. Intermediaries perform activities to 
reduce requirements for searching, transporting, and storing of goods or assets. They may also reduce the time from order to delivery. Customers benefit from saving costs and time in waiting and searching [8].

Value capture is about how digital innovators generate revenue streams, and how they get paid [36]. Examples of digital revenue creation are advertising, fees for usage and subscriptions, or charges for brokering services [25]. Credit card firms or operators of automated payment solutions can act as partners to transfer the values.

Not every digital company disrupts conventional business systems and remodels complete industries, like Amazon, Google, or Uber have done [41] p. 7. Digital innovation does not mean that a business system must be entirely newly created or be revolutionarily changed; the emphasis is on new combinations. Innovation comes from holistic thinking about the existing business model, and changing at least one dimension.

\section{Digital Innovators in Practice}

The following examples from various industries reflect different ways how digital innovators mix the ingredients. Networks are essential, other innovation components vary in the combinations. In every case, customers benefit from digital novelties.

\section{Learning Machines in Industrial Processes at Bosch Rexroth}

The German engineering company Bosch Rexroth produces hydraulics, electric drives and controls, assembly technologies, and more. Production processes at Bosch Rexroth can be optimized considerably with the aid of sensors and artificial intelligence. Digital capabilities are automatically built out of the processes. Machines in industrial applications start learning; they adjust internal parameters for self-improvement. Algorithms process recorded data and construct models for predictions with unknown data. The best times for maintenance activities on production machines are computed this way. Consequently, production downtimes and maintenance efforts are reduced to a minimum [39] pp. 345-349.

Tracking and tracing things as they flow though the various production stages is mature. However, further development of sensors, access technologies, and smart micro-devices will contribute to continuous improvements in production processes. Optimal reordering and refilling of supplies result in lower inventories. These improvements in asset management and production processes are cost-control measures that are hard for competitors to imitate [11] p. 43

Cost efficiency is the primary goal of digitization in industrial production [39] p. 13. However, not only process costs but also process flow times decrease. Because processes relate to products, customers' benefits are maximized as well: lower costs and shorter wait times for product deliveries [1] pp. 8-10.

\section{Customer Experience at Burberry}

Burberry, an apparel manufacturer from the United Kingdom, expanded their business by using intelligent technologies and by enhancing the customer experience in their retail stores. Burberry's offering addressed customers' needs seamlessly online and in stores [4]. Burberry introduced radio frequency identification (RFID), smart tablets, and large video projections at physical stores to display live videos, which adapt to fashion products that customers select for potential purchase [41] p. 9. Moreover, Burberry partnered with Google to develop a technology for lip detection [41] p. 8, which is enabled by artificial learning and neural network methods [9]. Users can send messages by kissing the display on a smartphone. The message receiver is notified that a Burberry kiss has been delivered. In both ways, Burberry improved the value type customer intimacy. Customers benefit from the brand image, relationship, and shopping experience.

\section{Vehicle Sharing}

Sharing of cars, bicycles, and e-scooters is constantly evolving in large cities. For example, Car2Go and DriveNow are car-sharing services founded by BMW and Daimler, the Mercedes manufacturer. These services make use of connectivity and the sharing trend in society. The car rental business system has been modified for higher service output. Vehicles are available at public points, for example, squares and roads. Smartphone apps are used for reservations, locating, hand-over (opening and locking), and invoicing. After usage, the cars can be left where they are. Compared to established car rental processes, customers save a significant amount of time picking up and returning vehicles. The ways in which value is captured are also noteworthy. Usage-based charges based on time or distance are more accurate than weekly or daily rates at traditional car rental agencies. In the future, additional service options may spontaneously be reserved via voice control, immediately provided, and charged based on usage, for example, navigation services, drive assistance systems, music streams, or additional (horse)power for higher speeds. 


\section{Remote-Controlled Health Care}

Increased health awareness in combination with miniaturized intelligent sensors on human bodies provide methods for various digital services. Medical measurements, such as blood pressure, heart rate, and blood-sugar level, can be monitored remotely at connected doctors' offices or hospitals. Elevations about individual thresholds can send early-warning messages or trigger alerts for medical treatment or emergency action. Implemented or carried medical devices, for example, insulin pumps and pacemakers, can be monitored externally via networks for functional continuity, which is vital. Robotic surgery is the most sophisticated discipline in digital health professions. It allows surgeons to operate via networks at a distance from the patient [22]. Thus, patients do not need to travel to a specialized surgeon who can be based anywhere in the world; they approach the nearest robot. Customers (patients) benefit from saving time and costs.

The Aravind Eye Hospital in Madurai (India) tested an Al system for early detection of signs of diabetic retinopathy. This illness can cause blindness if medically untreated. The Al system from Google uses neural networks and learns by evaluating large volumes of data. As a result, the scan and diagnose process takes only a few seconds so that many more people can be screened [21]. The automated and streamlined process corresponds to the customer value discipline operational excellence [42]. Benefits relate to lower costs and less time.

\section{Digital Methods for Insurance Companies}

Insurance companies can make great use of sensor data from health care and driver monitoring. Together with information that customers leave on networks, data analytics delivers individual risk profiles. Prices for health insurance policies can depend on current health condition, consumption behaviors, exercise, smoking/non-smoking status, etc. Prices for car insurance can depend on actual mileage, and on evaluated driving behavior. Price discounts can be offered to customers with lower risk profiles, which would be an economic benefit to them.

Insurance companies offer new services via chatbots, a kind of virtual, intelligent assistant that learns from user behavior and historical speech inputs. Chatbots are knowledgeable artificial consultants; they interact with customers like humans and provide specific answers in text format, regarding insurance offerings, conditions, and processes [32]. Users receive answers quickly and save time for searching.

Allianz Insurance tested a smartphone app with which pregnant women can monitor the heartbeat of their babies. The app can answer health questions and offers tailored insurance policies [20]. In this case, Allianz concentrates on customer benefits from relationships.

\section{Value Capture at Transport for London}

Transport for London (TfL) operates the subway and various public transportation services in Greater London. They improved their value capture by introducing the Oyster smartcard service based on near-field communication technology. Passengers do not need to understand the complex tariff systems of the London Underground and do not need to buy paper tickets; they just enter the subway with a contact-less read of their credit card. TfL included VISA as an intermediary for passenger authentication and for collection of payments [10]. Customers benefit from time savings and increased convenience.

\section{Digital Innovations to Extend Traditional E-Commerce}

Traditional e-commerce concerns spot market transactions of goods and services via the Internet. Businesses and consumers may be the buyer or the seller. E-commerce comprises both directions of value flows, good/services from the seller to the buyer, and secure payments in return. All phases and facets of the selling and buying process are considered, including technologies, solutions, features, and organization [24] pp. 383-424. The main application components are sales and promotion, procurement, logistics, production, financing and payments, and customer service [2] p. 38. The Internet serves as a tool to make the deal; it may also be part of the service delivery, for example, as medium for transmitting video streams or for monetary flows. The conventional e-commerce model is straightforward: It focusses on buyer and seller transactions and therefore, is constrained in terms of value delivery. E-commerce deals are highly efficient, and most importantly, provide cost advantages to suppliers and customers [28]. Digital innovators also provide customer value from collecting and evaluating sensor data, business model innovations, etc. The transformation from e-commerce to digital commerce relies upon the exploitation of digital data streams, that is, the continuous flow of digital codes generated by humans or machines. New information services can be offered to users by gathering and processing of digital data streams [28].

Users leave a huge amount of data for commercial evaluation. Data are aggregated from e-commerce transactions, from postings on social networks, and from other information people leave on the world wide web. Furthermore, the variety of connected sensors and user devices generates an incredible amount of data from sending alerts, syncing data, requesting updates, and monitoring of assets and people. This big data is centrally collected and evaluated. 
Changes in customer behaviors can be detected; products and services may, accordingly, be enhanced and/or tailored to particular needs. Customer satisfaction increases as a result [19]. Therefore, big data analytics may be viewed as an improvement function in the feedback loop of existing products or services. Analyses of big data also provide superior opportunities to invent new ways for creating value with novel products or distinctive business models [12].

The notion of cloud computing is another popular buzzword in the context of digital transformation. Although cloud is as nebulous as its name, it is an enduring trend in the industry. If cloud is understood as outsourcing of compute, storage, and applications services from shared data centers, then it may save costs, due to better resource utilization enabled by virtualization technologies [15]. These cost advantages provide value to the organization, as higher margins, and/or value to end customers, who pay a lower price.

The conventional transaction-based e-commerce model and its extension to mobile end-user devices (m-commerce) will continue. However, the model's functional evolution is limited in the current shape, if the demonstrated opportunities from digitization are not seized. Digital innovators and e-commerce traders use the Internet as the core for customer connectivity; the opportunity for e-commerce development is the use of continuous digital data streams via the Internet from and to customers. The traditional e-commerce model can evolve by considering the ideas presented on digital innovation and by answering the following questions. How can changes in the business model better meet customers' needs? How can the customer experience be increased? What technologies are advantageous for that purpose? What intermediaries may be included to increase the service outputs? Which social trends play a role? What kind of customer data is helpful? How can this data be gathered (smartphone app, sensors or transmitters on a customer's asset or body, chatbot, etc.)?

\section{Conclusions}

Industries and businesses are insecure regarding digitization; they are afraid of digital entrants that shake up established markets. This fear stems not only from prominent digital disruptions but also from lack of awareness of the essentials of digitization. To present a more comprehensive view of digital innovations, we attempted to present the principal elements of digital innovations that foster business growth.

Digitization is not a hype; it is about utilizing technological progress mixed with business and sociocultural aspects. Firms that provide (digital) customer advantage will achieve competitive advantage as a result [45]. Firms that neglect digitization today will probably lose market share, and eventually, disappear from marketplaces. The conventional e-commerce model is old. Further e-commerce growth calls for enhancements from digital innovation, by continuously collecting and evaluating data from intelligent, sensor-equipped objects, to boost the customer experience.

Digital innovation puts the customer's benefits and values in focus. Starting from this point, new opportunities can be seized by connecting smart devices, modifying business systems, and/or exploiting social trends. Smart devices connected via the Internet or via private networks are the sense organs and the hearts of digital solutions. They allow collection of physical data, which is needed to provide appropriate digital services. Data is collected from many natural and artificial objects, and is permanently available, independent of location. Digital innovators evaluate and process this data, always keeping the customer value in mind.

The case studies presented showed a few of the numerous ways to include and mix digital ingredients. It is up to the digital innovator's creativity how to assemble the digital elements to meet special customers' needs. Businesses should not necessarily strive to turn an industry upside down, as so-called digital disruptors have demonstrated. Even slight modifications of business systems using connected smart devices can result in significant customer advantages.

We did not mention legislation and security, which is a limitation of the article. Legislation may slow down or even destroy digital business models. For example, the app UberPop, which brokers transportation services from private cars, has been banned in Germany, to protect the business of licensed taxicabs [37]. Security matters, notably in view of technology progress and use, for example, in relation to data privacy. Convenient and reliable methods for user authentication are at the center of research and development.

\section{References}

[1] R. Anupindi, S. Chopra, D. Deshmukh, J. van Mieghem, and E. Zemel, Managing Business Process Flows. Upper Saddle River, NJ: Prentice Hall, 1999.

[2] F. Aulkemeier, M. Schramm, M.-E. lacob, and J. van Hillegersberg, A service-oriented e-commerce reference architecture, Journal of Theoretical and Applied Electronic Commerce Research, vol. 11, no. 1, pp. 26-45, 2016.

[3] M. J. Baker and S. J. Hart, Product Strategy and Management, 2nd ed. Harlow, UK: Pearson, 2007.

[4] A. Blitz, Beset by the digital revolution successful retailers embrace technology that enhances customer value, Strategy \& Leadership, vol. 44, no. 6, pp. 16-24, 2016. 
[5] J. Bloomberg. (2018) Digitization, digitalization, and digital transformation: Confuse them at your peril. Forbes. [Online]. Available: https://www.forbes.com/sites/jasonbloomberg/2018/04/29/digitization-digitalization-anddigital-transformation-confuse-them-at-your-peril/\#567535a52f2c.

[6] K. Bredmar, Digitalisation of enterprises brings new opportunities to traditional management control, Business Systems Research, vol. 8, no. 2, pp. 115-125, 2017.

[7] E. Brynjolfson and A. McAfee, The Second Machine Age: Work, Progress, and Prosperity in a Time of Brilliant Technologies. New York, NY: Norton, 2016.

[8] A. T. Coughlan, E. Anderson, L. W. Stern, and A. I. El-Ansary, Marketing Channels, 6th ed. Upper Saddle River NJ: Prentice Hall, 2001

[9] J. A. Dargham, A. Chekima and S. Omatu, Lip detection by the use of neural networks, Artificial Life and Robotics, vol. 12, no. 1-2, pp. 301-306, 2008.

[10] B. Dobson. (n.d.) NFC in transport for London. NFC Forum. [Online]. Available: https://members.nfcforum.org/resources/presentations/Brian Dobson Transport for London.pdf.

[11] D. Dranove and S. Marciano, Kellogg on Strategy - Concepts, Tools, and Frameworks for Practitioners. Hoboken, NJ: John Wiley \& Sons, 2005.

[12] M. Huberty, Awaiting the second big data revolution: From digital noise to value, Journal of Industry Competition and Trade, vol. 15, no. 1, pp. 35-47, 2015

[13] F. Isada and Y. Isada, Network analysis of innovation in the Internet of Things, Interdisciplinary Description of Complex Systems: INDECS, vol. 16, no. 2, pp. 216-226, 2018.

[14] M. W. Johnson, C. M. Christensen and H. Kagermann, Reinventing your business model, Harvard Business Review, vol. 86, no. 12, pp. 50-59, 2008.

[15] V. Josyula, M. Orr and G. Page, Cloud Computing: Automating the Virtualized Data Center. Indianapolis, IN: Cisco Press, 2012.

[16] W. Kathan, K. Matzler and V. Veider, The sharing economy: Your business model's friend or foe?, Business Horizons, vol. 59, no. 6, pp. 663-672, 2016.

[17] D. Kellmereit and D. Obodovski, The Silent Intelligence: The Internet of Things. San Francisco, CA: DnD Ventures, 2013.

[18] A. Knoll, Wettlauf um künstliche Intelligenz: Wie kann Europa mithalten? Handelsblatt Journal, extra edition Future IT Management, pp. 18-19, 2017.

[19] I. Lee and K. Lee, The internet of things (loT): Applications, investments, and challenges for enterprises, Business Horizons, vol. 58, no. 4, pp. 431-440, 2015.

[20] A. Maier and D. Palan, Allianz goes Google, Manager Magazine, June, pp. 50-56, 2016

[21] C. Metz. (2019, March) India fights diabetic blindness with help from A.I. The New York Times. [Online]. Available: https://www.nytimes.com/2019/03/10/technology/artificial-intelligence-eye-hospital-india.html

[22] B. Morris. (2005, September) Robotic surgery: Applications, limitations, and impact on surgical education. Medscape General Medicine. [Online]. Available: https://www.ncbi.nlm.nih.gov/pmc/articles/PMC1681689/.

[23] A. Nadeem, B. Abedin, N. Cerpa, and E. Chew, Editorial: Digital transformation \& digital business strategy in electronic commerce - The role of organizational capabilities, Journal of Theoretical and Applied Electronic Commerce Research, vol. 13, no. 2, pp. I-VIII, 2018.

[24] J. A. O'Brien and G. M. Marakas, Management Information Systems, 10th ed. New York, NY: McGraw-Hill, 2010

[25] A. Osterwalder and Y. Pigneur, Business Model Generation: A Handbook for Visionaries, Game Changers, and Challengers. Hoboken, NJ: John Wiley \& Sons, 2010.

[26] P. Parviainen, M. Tihinen, J. Kääriäinen, and S. Teppola, Tackling the digitalization challenge: How to benefit from digitalization in practice, International Journal of Information Systems and Project Management, vol. 5, no. 1, pp. 63-77, 2017.

[27] A. Payne and P. Frow, A strategic framework for customer relationship management, Journal of Marketing, vol. 69, no. 4, pp. 167-176, 2005.

[28] F. Pigni, G. Piccoli and R. Watson, Digital data streams: Creating value from the real-time flow of big data, California Management Review, vol. 58, no. 3, pp. 5-25, 2016.

[29] M. E. Porter, Competitive Strategy. New York, NY: Free Press, 1980.

[30] M. E. Porter, Competitive Advantage. New York, NY: Free Press, 1985.

[31] U. Raza, P. Kulkarni and M. Sooriyabandara, Low power wide area networks: An overview, IEEE Communications Surveys \& Tutorials, vol. 19, no. 2, 2017.

[32] M. Riikkinen, H. Saarijärvi, P. Sarlin, and I. Lähteenmäki, Using artificial intelligence to create value in insurance, International Journal of Bank Marketing, vol. 36, no. 6, pp. 1145-1168, 2018.

[33] V. Roblek, M. Pejić Bach, M. Meško, and A. Bertoncelj, The impact of social media to value added in knowledge-based industries, Kybernetes, vol. 42, no. 4, pp. 554-568, 2013.

[34] M. Sawhney. (2003, July) The seven fundamentals of value (according to Mohanbir Sawhney). CIO. [Online]. Available: $\quad$ https://www.cio.com/article/2441854/the-seven-fundamentals-of-value--according-to-mohanbirsawhney-.html.

[35] M. Sawhney, G. Verona and E. Prandelli, Collaborating to create: The Internet as a platform for customer engagement in product innovation, Journal of Interactive Marketing, vol. 19, no. 4, pp. 4-17, 2005.

[36] M. Sawhney, R. C. Wolcott and I. Arroniz, The 12 different ways for companies to innovate, MIT Sloan Management Review, vol. 47, no. 3, pp. 75-81, 2006.

[37] P. Seibt. (2016), Uber verliert vor Gericht - und nun? Spiegel Online. [Online]. Available: https://www.spiegel. de/wirtschaft/unternehmen/fahrdienst-uber-uberpop-bleibt-in-deutschland-verboten-a-1096768.html. 
[38] T. Shamma. (2017, March) Google glass didn't disappear. You can find it on the factory floor. NPR. [Online]. Available: https://www.npr.org/sections/alltechconsidered/2017/03/18/514299682/google-glass-didnt-disappearyou-can-find-it-on-the-factory-floor?utm campaign=storyshare\&utm source=facebook.com\&utm medium=so cial?utm campaign=storyshare\&utm source=facebook.com\&utm medium=social\&t=1556475737845

[39] D. Slama, F. Puhlmann, J. Morrish, and R. M. Bhatnagar, Enterprise IOT: Strategies \& Best Practices for Connected Products \& Services. Sebastopol, CA: O'Really Media, 2015.

[40] D. Suša Vugec, K. Tomičić-Pupek and V. Bosilj Vukšić, Social business process management in practice: Overcoming the limitations of the traditional business process management, International Journal of Engineering Business Management, vol. 10, pp. 1-10, 2018.

[41] T. Szigeti, D. Zacks, M. Falkner and S. Arena, S., Cisco Digital Network Architecture: Intent-based Networking for the Enterprise. Indianapolis, IN: Cisco Press, 2019.

[42] M. Treacy and F. Wiersema, The Discipline of Market Leaders. Choose Your Customers, Narrow Your Focus, Dominate Your Market. Reading, MA: Addison-Wesley, 1995.

[43] P. Trott, Innovation Management and New Product Development, 6th ed. Harlow, UK: Pearson, 2017.

[44] G. L. Urban and J. R. Hauser, Listening in to find and explore new combinations of customer needs, Journal of Marketing, vol. 68, no. 2, pp. 72-87, 2004.

[45] R. B. Woodroff, Customer value: The next source for competitive advantage, Journal of the Academy of Marketing Science, vol. 25, no. 2, pp. 139-153, 1997. 